\title{
Lumbar Facet Joint Synovial Cysts: Does T2 Signal Intensity Predict Outcomes after Percutaneous Rupture?
}

\author{
S.C. Cambron, J.J. McIntyre, S.J. Guerin, Z. Li, and D.A. Pastel
}

\begin{abstract}
BACKGROUND AND PURPOSE: Lumbar facet synovial cysts are a cause of back pain and radiculopathy with facet joint degeneration, the most common cause for cyst formation. Typically, LFSCs are T2 hyperintense on MR imaging, but the signal intensity is variable. Treatment options include percutaneous rupture and surgical resection. This study evaluates the relationship between LFSC signal intensity on MR imaging and outcomes as it relates to percutaneous rupture success and need for subsequent surgery.
\end{abstract}

MATERIALS AND METHODS: A retrospective review of 110 patients who underwent CT fluoroscopic-guided rupture of symptomatic LFSCs was performed. The LFSCs were characterized by their T2 signal intensity on MR imaging and divided into 3 groups: high, intermediate, and low T2 signal intensity. The rates of successful cyst rupture and need for subsequent surgery were recorded.

RESULTS: Percutaneous LFSC rupture was technically successful in $87 \%$ of all cases. Cyst rupture was successful in $89 \%$ and $90 \%$ of high and intermediate signal intensity cysts, respectively, and in $65 \%$ of low signal intensity cysts $(P=.017, .030)$. High signal intensity cysts had lower postprocedural surgical rates (29\%) when compared with intermediate and low signal cyst as a group $(P=.045)$.

CONCLUSIONS: T2 hyperintense and intermediate signal intensity LFSCs are easier to rupture, perhaps because the cysts contain a higher proportion of fluid and are less gelatinous or calcified than T2 hypointense cysts. Patients with T2 hyperintense LFSCs are less likely to need surgery.

ABBREVIATIONS: LFSC = lumbar facet synovial cyst

S ynovial cysts arising from the lumbar spine facet joints are most commonly seen in the setting of degenerative spondylosis of the facet joints. ${ }^{1-6}$ The incidence of lumbar facet synovial cysts detected by imaging ranges from $0.8-2.0 \%{ }^{7}$ LFSCs most commonly arise at the L4-L5 spinal level. ${ }^{7}$ On MR imaging, synovial cysts are typically T2-hyperintense and T1-hypointense, but the cyst signal is variable, depending on protein content, previous hemorrhage, and calcification. ${ }^{7}$

LFSCs that project into the spinal canal can cause radiculopathy $(87 \%)$, neurogenic claudication $(44 \%)$, sensory loss $(43 \%)$, and motor weakness (27\%). ${ }^{8}$ Treatment options include surgical resection and percutaneous rupture. Percutaneous rupture of

\footnotetext{
Received August 7, 2012; accepted after revision October 20.

From the Department of Radiology (S.C.C., D.A.P., J.J.M., S.J.G.), Section of Neuroradiology (J.J.M., S.J.G.), and Quantitative Biomedical Science (Z.L.), Dartmouth Hitchcock Medical Center, Lebanon, New Hampshire.

Paper previously presented at: Annual Meeting of the American Society of Neuroradiology, April 26, 2012; New York, New York.

Please address correspondence to Dr. Sword C. Cambron, Department of Radiology, Dartmouth Hitchcock Medical Center, Lebanon NH, One Medical Center Dr, Lebanon, NH 03766; e-mail: swordrads@gmail.com

http://dx.doi.org/10.3174/ajnr.A3441
}

LFSCs is most commonly performed indirectly through the facet joint by use of radiography or CT fluoroscopy. A retrospective study by Allen et $\mathrm{al}^{6}$ studied 32 patients and reported excellent long-term pain relief in $72 \%$ of patients undergoing percutaneous facet cyst rupture. The largest report of nonoperative treatment for lumbar facet joint synovial cysts by Martha et $\mathrm{al}^{9}$ followed 101 patients after percutaneous cyst rupture with epidural steroid injection. They found that $46 \%$ of patients achieved permanent symptomatic relief with percutaneous therapy.

To date, no studies have evaluated the relationship between cyst signal intensity on MR imaging and outcomes after percutaneous rupture. It is possible that cysts of certain signal intensities are more difficult to rupture and more likely to require surgical intervention to relieve symptoms. This study evaluates the relationship between LFSC signal intensity on MR imaging and outcomes in 110 patients as it relates to percutaneous rupture success and the need for subsequent surgery.

\section{MATERIALS AND METHODS}

We retrospectively reviewed the clinical outcome and imaging of 110 patients with 113 LFSCs who underwent attempted CT- 

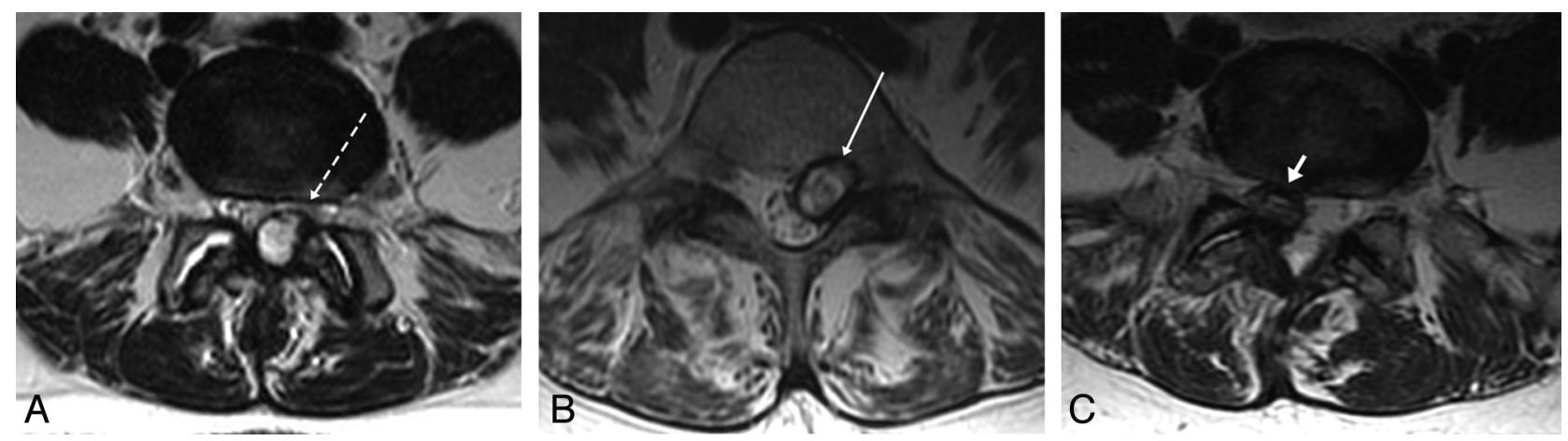

FIG 1. Example of 3 different synovial cysts with 3 different signal intensities. A, High-signal left-sided synovial cyst (dashed arrow). B, Left-sided intermediate signal cyst (arrow). C, Right-sided low signal cyst (arrowhead).

guided synovial cyst rupture as primary treatment for symptomatic LFSCs. The Institutional Review Board at Dartmouth Hitchcock Medical Center waived the consent for this review. Inclusion criteria consisted of lower-extremity radiculopathy corresponding to the location of a LFSC and MR imaging within 2 months before initial percutaneous rupture. A chart review was performed with the following data collected: patient demographics, cyst signal intensity, size and location, rupture success, number of repeat procedures, and need for subsequent surgical resection. If surgery was performed, the level, side, and type of surgery were recorded.

The diagnosis of LFSC was established on lumbar spine MRI. T2 axial and sagittal images were obtained for all study patients with the following parameters: TR of 3000-6000 msec, TE of $80-110 \mathrm{msec}$, section thickness of 4-mm with 5-mm spacing, and a matrix of $256 \times 256$ or $512 \times 512$.

The signal intensity of each synovial cyst on preprocedure MR imaging was reviewed independently by a second-year radiology resident and a neuroradiology faculty member. In cases in which there was disagreement, a third attending neuroradiologist reviewed the imaging and determined the cyst signal intensity. LFSCs were divided into 3 groups, on the basis of T2 signal intensity. High signal intensity cysts were CSF isointense or hyperintense, intermediate signal intensity cysts were CSF hypointense but muscle hyperintense, and low signal intensity cysts were muscle isointense or hypointense (Fig 1). When cyst signal intensity was mixed, the predominant signal was used. The signal intensity of a cyst on a repeat procedure was only included in the analysis if a repeat MR imaging was done within 2 months before the repeat procedure. Synovial cyst size was measured in each case on axial images, measuring from the outer wall of the hypointense cyst rim to the opposite outer wall. Cyst wall thickness was not evaluated.

Likelihood ratio $\chi^{2}$ and Fisher exact test were used to examine the associations between signal intensity, procedural success, and surgical rates.

All procedures were performed between January 2004 and December 2011 by 7 different neuroradiologists performing 1-56 procedures. Most the procedures were performed by 3 neuroradiologists. During the procedure, moderate sedation was administered and $1 \%$ lidocaine buffered with sodium bicarbonate was used for local anesthesia. A 20-ga spinal needle was then advanced under intermittent CT fluoroscopy into the facet joint and 1-2 $\mathrm{mL}$ of Omnipaque 350 (iohexol; Nycomed, Princeton, New Jer- sey) diluted in normal saline at a ratio of 1:10 was injected to confirm continuity of the facet joint with the synovial cyst. In patients with osteophytes covering the facet joint, a 14-ga coaxial system (Bonopty; AprioMed, Uppsala, Sweden) was used to access the facet joint. Once the cyst was opacified with contrast, a high-pressure syringe was used to rupture the cyst into the epidural space by use of dilute contrast. A technically successful cyst rupture was determined by the loss of resistance method and by extravasation of contrast outside the confines of the cyst wall on CT. After cyst rupture, $80 \mathrm{mg}$ of methylprednisolone (DepoMedrol; Pfizer, New York, New York) was injected. Patients were observed for 1 hour after the procedure.

\section{RESULTS}

One hundred ten patients with 113 individual LFSCs underwent 153 procedures. One patient was treated at 2 levels, and 2 patients were treated bilaterally. Twenty-five patients were treated multiple times at the same level and side for recurrence of their symptoms. The number of repeat procedures ranged from $1-5$, for a total of 40 repeat procedures, and time to repeat ranged from 2-800 days, with a mean of 162 days. Preprocedure MR imaging was available in 21 of the 40 repeat procedures, allowing us to evaluate 134 synovial cysts before rupture (Table 1).

The mean patient age was 63 years, and most were female (65\%). The most common level treated was L4-L5 (66\%); 87\% of all LFSCs were ruptured successfully. The maximum number of repeat attempts on a single patient was 5 . Follow-up ranged from 7-93 months after the last attempted percutaneous rupture, with a mean follow-up of 34 months; $43 \%$ of patients eventually had surgery at mean time of 183 days after last attempted rupture.

We found 54 high signal LSFCs, 63 intermediate signal LSFCs, and 17 low signal LSFCs.

Mean axial cyst size was $10.6 \mathrm{~mm}$, with the largest cyst measuring $18 \mathrm{~mm}$ and the smallest $5 \mathrm{~mm}$. Cyst size did not affect signal intensity, rupture rate, or surgical rate.

The rate of successful LFSC rupture was calculated for each group (Table 2). High and intermediate signal intensity cysts were successfully ruptured at high rates ( $89 \%$ and $90 \%$, respectively) and were significantly easier to rupture than low signal intensity cysts $(P=.017, .030)$.

Twenty-nine percent of patients identified with high signal intensity cysts on initial imaging had surgical treatment. This sur- 
Table 1: Descriptive statistics of the study sample

\begin{tabular}{|c|c|}
\hline Total number of patients & 110 \\
\hline $\begin{array}{l}\text { Total number of individual } \\
\text { LFSCs }\end{array}$ & 113 \\
\hline Age, y & Mean, 63 (SD, 11; range, 28-87) \\
\hline Sex, $n(\%)$ & Female, $71(65)$ \\
\hline \multicolumn{2}{|l|}{ Cyst level, $n(\%)$} \\
\hline L2-L3 & $6(4.5)$ \\
\hline L3-L4 & $17(12.7)$ \\
\hline L4-L5 & $89(66.4)$ \\
\hline L5-S1 & $22(16.4)$ \\
\hline Follow-up period, mo & $\begin{array}{l}\text { Mean, } 34(n=110 ; S D, 24.5 \\
\text { range, 7-93) }\end{array}$ \\
\hline Successful rupture, $n(\%)$ & $116(86.6)$ \\
\hline Subsequent surgery, $n(\%)$ & $44(39)$ \\
\hline Time to first surgery, $d$ & Mean 183 (SD 226, range 4-1279) \\
\hline \multicolumn{2}{|l|}{ Repeat procedures, $n(\%)$} \\
\hline None & $88(78)$ \\
\hline 1 & $16(14)$ \\
\hline 2 & $6(5)$ \\
\hline$\geq 3$ & $3(3)$ \\
\hline Total additional procedures, $n$ & 40 \\
\hline $\begin{array}{l}\text { MRI before repeat procedure, } \\
n(\%)\end{array}$ & $21(52)$ \\
\hline $\begin{array}{l}\text { Mean days to repeat } \\
\text { procedure }\end{array}$ & $\begin{array}{l}162(\text { median }=71, \operatorname{Max}=800, \\
\quad \operatorname{Min}=2)\end{array}$ \\
\hline Total procedures, $n$ & 153 \\
\hline $\begin{array}{l}\text { Total procedures with prior } \\
\text { MRI, } n\end{array}$ & 134 \\
\hline
\end{tabular}

Table 2: Signal intensity and procedural success

\begin{tabular}{lcccc}
\hline Signal Intensity & $\begin{array}{c}\text { Success, } \\
\boldsymbol{n}(\%)\end{array}$ & $\begin{array}{c}\text { Unsuccessful, } \\
\boldsymbol{n}(\%)\end{array}$ & $\begin{array}{c}\text { Total } \\
\text { Value }\end{array}$ \\
\hline High & $48(89)$ & $6(11)$ & 54 & .03 \\
Intermediate & $57(90)$ & $6(10)$ & 63 & .017 \\
Low & $11(65)$ & $6(35)$ & 17 & \\
Total & $116(87)$ & $18(13)$ & 134 & \\
\hline
\end{tabular}

Table 3: Signal intensity and surgery

\begin{tabular}{lcccc}
\hline \multicolumn{1}{c}{ Signal } & $\begin{array}{c}\text { No } \\
\text { Surgery, } \\
\boldsymbol{n}(\%)\end{array}$ & $\begin{array}{c}\text { Surgery, } \\
\boldsymbol{n}(\%)\end{array}$ & Total & $\begin{array}{c}\boldsymbol{P} \\
\text { Value }\end{array}$ \\
\hline High & $34(71)$ & $14(29)$ & 48 & \\
Intermediate and low & $34(52)$ & $31(48)$ & 65 & \\
Total & $68(60)$ & $45(40)$ & 113 & .045 \\
\hline
\end{tabular}

Table 4: Repeat rupture and surgery

\begin{tabular}{lcccc}
\hline $\begin{array}{c}\text { Repeat } \\
\text { Procedure }\end{array}$ & $\begin{array}{c}\text { No Surgery, } \\
\boldsymbol{n}(\%)\end{array}$ & $\begin{array}{c}\text { Surgery, } \\
\boldsymbol{n}(\%)\end{array}$ & Total & $\begin{array}{c}\boldsymbol{P} \\
\text { Value }\end{array}$ \\
\hline No & $54(61)$ & $34(39)$ & 88 & \\
Yes & $14(56)$ & $11(45)$ & 25 & \\
Total & $68(60)$ & $45(40)$ & 113 & .63 \\
\hline
\end{tabular}

gical rate is significantly less $(P=.045)$ than in patients with intermediate and low signal intensity cysts (48\%) identified on initial imaging (Table 3 ).

In most patients (23/25) who underwent repeat percutaneous procedures, the cyst successfully ruptured during the initial procedure; $45 \%$ of patients with repeat attempted cyst rupture eventually had surgery. This is not significantly different from the $39 \%$ surgical rate in patients who underwent a single attempted rupture $(P=.63)$ (Table 4$)$.

\section{Complications}

Two LFSCs ruptured into the subarachnoid space, and steroid was not given. One patient had increased pain and underwent surgical decompression 4 days later. The procedure was not complicated by infection or hemorrhage in any case.

\section{DISCUSSION}

This case series is the first to report the relationship between T2 signal intensity of LFSCs on MR imaging with percutaneous rupture rate as a primary outcome. To our knowledge, this study is the largest (110 patients) clinical series to evaluate the nonsurgical care of LFSCs. The patient demographics (65\% female) and distribution of treated cysts ( $66 \%$ at the L4-L5 level) are similar to previous publications. ${ }^{9,10}$ Most treatment attempts (87\%), as defined by visualization of contrast outside the confines of the cyst wall on CT, were successful, but the success rate varied, depending on MR imaging signal intensity of the cyst. High and intermediate signal intensity cysts are significantly easier to rupture than low signal intensity cysts. The reason for this is not entirely clear, but one possible explanation is that elevated protein content and/or previous hemorrhage in the low signal intensity cysts results in viscous contents making cyst rupture more difficult. ${ }^{7}$ Another explanation is partial or complete calcification of the cyst, which was not evaluated in this study. Knowledge of the MR imaging signal intensity of the synovial cyst before percutaneous treatment can be helpful in counseling patients with symptomatic LFSCs.

The need for subsequent surgical intervention was evaluated as a secondary outcome. We found that percutaneous rupture with steroid injection allowed $60 \%$ of all patients to avoid surgery. This rate is higher than the $46 \%$ rate reported by Martha et $\mathrm{al}^{9}$ and the $55 \%$ rate reported by Allen et al. ${ }^{6}$ We found a significantly lower rate of surgery in patients with high signal intensity cysts (29\%) when compared with intermediate and low signal cysts as a group $(P=.045)$.

A minority of patients underwent repeat LFSC rupture in our population, and no significant difference was seen in this subgroup with regard to subsequent surgery. We did not find a distinguishing characteristic that determined need for repeat rupture because most cysts were ruptured initially and the signal characteristics of these LFSCs were not significantly different.

This study has limitations, one of which is the lack of pretreatment and posttreatment pain and disability scores. Our reliance on subsequent surgery as a secondary outcome measure does not take into account those patients who are unable to, or elect not to, have surgery despite continuing or worsening pain. Because follow-up consisted of chart review, our surgical rate reflects surgical data at our institution. It is likely that some patients sought surgery at an outside institution. Our findings are also limited by the fact that the data were collected over a range of follow-up periods (7-93 months). Patients enrolled toward the end of the study may still have surgery for LFSCs. Future research could improve on this study with a large-scale prospective study with the use of pain and disability scores.

\section{CONCLUSIONS}

Most patients with symptomatic LFSCs can avoid surgery with percutaneous rupture and steroid injection regardless of cyst size and signal intensity on MR imaging. Patients with T2-hyperin- 
tense synovial cysts are most likely to benefit from nonsurgical intervention. This information is valuable when counseling patients on their treatment options for symptomatic LFSCs.

\section{REFERENCES}

1. Abdullah AF, Chambers RW, Daut DP. Lumbar nerve root compression by synovial cysts of the ligamentum flavum. J Neurosurg 1984;60:617-20

2. Brish A, Payan HM. Lumbar intraspinal extradural ganglion cyst. J Neurol Neurosurg Psychiatry 1972;35:771-75

3. Kao CC, Uihlein A, Bickel WH, et al. Lumbar intraspinal extradural ganglion cyst. J Neurosurg 1968;29:168-72

4. Kurz LT, Garfin SR, Unger AS, et al. Intraspinal synovial cyst causing sciatica. J Bone Joint Surg Am 1985;67:865-71

5. Alicioglu B, Sut N. Synovial cysts of the lumbar facet joints: a retro spective magnetic resonance imaging study investigating their relation with degenerative spondylolisthesis. Prague Medical Report 2009;110:301-09

6. Allen TL, Tatli Y, Lutz GE. Fluoroscopic percutaneous lumbar zygapophyseal joint cyst rupture: a clinical outcome study. Spine $J$ 2009;9:387-95

7. Apostolaki E, Davies AM, Evans N, et al. MR imaging of lumbar facet joint synovial cysts. Eur Radiol 2000;10:615-23

8. Pytel P, Wollmann RL, Fessler RG, et al. Degenerative spine disease pathologic findings in $\mathbf{9 8 5}$ surgical specimens. Am J Clin Pathol 2006;125:193-202

9. Martha JF, Swaim B, Wang DA, et al. Outcome of percutaneous rupture of lumbar synovial cysts: a case series of 101 patients. Spine J 2009;9:899-904

10. Doyle AJ, Merrilees M. Synovial cysts of the lumbar facet joints in a symptomatic population: prevalence on magnetic resonance imaging. Spine 2004;29:874-78 